\title{
CARACTERIZACIÓN DE LAS CONSTRUCCIONES PASIVAS CON COMPLEMENTO AGENTE EXPLÍCITO
}

\author{
CHARACTERIZATION OF PASSIVE \\ CONSTRUCTIONS WITH EXPLICIT \\ AGENT COMPLEMENT
}


En el marco teórico del Enfoque Cognitivo-Prototípico, el presente trabajo propone el estudio comparativo de las construcciones pasivas con complemento agente (CAg) explícito introducido por las preposiciones por y de en discurso auténtico. Se sostiene que en el caso de la preposición de, el atributo más relevante para su aparición en contexto de CAg es el tipo de predicación: de ocurre con predicaciones estáticas, con pacientes poco afectados, más allá del grado de determinación del agente y paciente, y de si son [+humanos] o [-humanos]. La preposición por, en contraste, ocurre en predicaciones dinámicas, con pacientes altamente afectados. El hecho de que la preposición de sea elegida por el hablante en las predicaciones estáticas se debe al significado básico de posesión que tiene de (Funes, 2015). Para comprobar las hipótesis, se analizarán ejemplos del corpus oral PRESEEA-Buenos Aires (Proyecto para el Estudio Sociolingüístico del Español de España y de América), de los años 2010-2011 y de un corpus escrito de notas periodísticas del año 2014.

Palabras Clave: construcciones pasivas, complemento agente, preposición de, preposición por

This paper shows the results of a comparative study on passive constructions with explicit agent complement $(\mathrm{AgC})$ introduced by prepositions por and $d e$ in authentic contexts, analyzed within the framework of the Prototypical-Cognitive Approach. Preposition de occurs in static predications, with low affected patients, no matter the degree of determination of the agent and the patient, or if they are [+human] or [-human]. By contrast, preposition por occurs in dynamic predications with highly affected patients. The fact that preposition $d e$ is chosen in static predications is due to its basic meaning of possession (Funes, 2015). The analysis is based on different examples taken from an oral corpus (PRESEEABuenos Aires, 2010-2011) as well as a written corpus (2014).

KEY wORDS: passive constructions, agent complement, preposition $d e$, preposition por

RECEPCIÓN: 26/06/2015

ACEPTACIÓN: 16/09/2015 


\section{CARACTERIZACIÓN DE LAS CONSTRUCCIONES PASIVAS CON COMPLEMENTO AGENTE EXPLÍCITO}

\section{CHARACTERIZATION OF PASSIVE CONSTRUCTIONS WITH EXPLICIT AGENT COMPLEMENT}

María Soledad Funes Universidad de Buenos Aires

\section{Introducción}

El presente trabajo describe comparativamente las construcciones pasivas con complemento agente $(\mathrm{CAg})$ explícito introducido por las preposiciones por y de (como por ejemplo, en maltratada por él y rodeado de amor) con el objetivo de ofrecer una caracterización sistemática de ambas construcciones y explicar las motivaciones que llevan al hablante a elegir una u otra preposición para encabezar la función de CAg. Se parte del presupuesto teórico del Enfoque Cognitivo Prototípico (ECP) de que la Sintaxis está motivada por la Semántica y la Pragmática, es decir, todo uso de una forma es significativo y responde a los objetivos comunicativos de los hablantes (Langacker, 1987; 1991; Lakoff, 1987, entre otros). Desde el ECP, el valor de uso de las formas se explica a partir de sus contextos de aparición, medidos en forma cualitativa y cuantitativa. Asimismo, se consideran los resultados de trabajos previos (Funes, 2011; 2012). En 
un primer trabajo (Funes, 2010, publicado en 2012) se caracterizó el CAg introducido por la preposición de, comparándolo con los atributos del agente y del paciente de la cláusula transitiva prototípica (Hopper y Thompson, 1980). ${ }^{1}$ En un trabajo posterior (Funes, 2011), se estudió la alternancia de las preposiciones de y por como introductoras del CAg y se concluyó que el hablante elige la preposición por para acompañar agentes con los atributos [+humano] y [+determinado], en contextos de pacientes con media o alta afectación, y en esquemas de predicaciones dinámicas (manifestadas en construcciones pasivas de frase verbal con ser o predicaciones no finitas). En contraste, el hablante elige de para acompañar agentes con el atributo [-humano] y [-determinado], en contextos de sujetos pacientes con baja afectación, y en esquemas de predicaciones con entidades estáticas (manifestadas mayormente por predicaciones no finitas).

Considerando los resultados previos, el presente trabajo espera aportar nueva evidencia empírica respecto de la alternancia entre las preposiciones por y de como introductoras del CAg. Se reforzará el análisis con una nueva hipótesis, que plantea que existe una jerarquía en los atributos de las construcciones. Se sostiene que en la elección de una preposición o de otra para introducir el CAg, el atributo que más

\footnotetext{
${ }^{1}$ Se denomina cláusula transitiva prototípica (CTP) a la gramaticalización del modelo cognitivo idealizado de bola de billar, según el cual un agente transmite energía y provoca un cambio considerable en el paciente, como por ejemplo en Juan rompió el vidrio. Este traspaso de energía que provoca una modificación en el segundo actante es lo que las gramáticas han denominado transitividad. Sin embargo, para el ECP, la transitividad no es una característica del verbo sino del mensaje, de la cláusula (Hopper y Thompson, 1980: 251).
} 
pesa es el tipo de predicación: el hablante elige de en contextos de predicaciones estáticas, con pacientes poco afectados, más allá del grado de determinación del agente y paciente, y de si es [+humano] o [-humano]. El hecho de que la preposición de sea elegida por el hablante en las predicaciones estáticas se relaciona con el significado básico que tiene de, que es el de posesión (Funes, 2015). En tanto, el hablante elige la preposición por en contextos de predicaciones dinámicas, donde se observa la correlativa alta afectación del paciente.

En función de las exigencias del marco teórico, para probar las hipótesis, se analizarán cuantitativa y cualitativamente ejemplos provenientes del corpus oral PRESEEABuenos Aires (2010-2011) y un corpus escrito del año 2014.

Cabe aclarar que se relevó la presencia de CAg introducido por la preposición por y por de en contextos de sentido pasivo, tanto de voz pasiva de frase verbal con ser como de construcción pronominal con se, en contextos de estar + atributo $^{2}$ y en predicaciones no finitas. ${ }^{3}$

\footnotetext{
${ }^{2}$ Con respecto a la existencia de sentido pasivo en las construcciones con verbo estar, véanse, entre otros: RAE (1973: §3.12.8.), Rosetti (1969: §5.3.), Seco (1999: $\$ 8.8 .5$.), Delbecque (2003) y RAE-ASALE (2009: $\$ 28.16$ d y e).

${ }^{3}$ Se denomina predicación no finita a aquella que no tiene verbo, pero que es tratada como predicación tanto por la semántica eventiva de la base del participio como por asociar dos actantes con roles claros (en términos de la cláusula transitiva prototípica) y en el caso de las construcciones pasivas tratadas aquí, explícitos (agente y paciente presentes en la cláusula). Al respecto de este tipo de construcciones, la RAE-ASALE (2009) reconoce el sentido pasivo de oraciones como Una novela leída por todo el mundo, a las que llama "oraciones pasivas sin verbo 'ser'”, y aclara que para algunas gramáticas estas estructuras se denominan "relativas reducidas" (2009: $\$ 41.2 b)$.
} 


\section{Estado de la cuestión}

\subsection{Las construcciones pasivas}

\subsubsection{Las gramáticas hispánicas}

En las gramáticas hispánicas, se observan distintos enfoques en el estudio de las construcciones pasivas (CP). La Gramática de la Real Academia Española (GRAE) de 1931 (\$83) y Alonso/H. Ureña (1938-1939: \$128) tienen un enfoque morfológico, es decir, analizan el cambio de voz en español como un accidente o morfema verbal que señala si la significación del verbo es producida o recibida por el sujeto. Bello (1980[1847]: \$430), Alcina Franch/Blecua (1975: $\$ 5.4 .1)$, Rosetti (1969: 72), Kovacci (1990-1992: 69), Mendikoetxea (1999) y la Nueva gramática de la lengua española (RAE-ASALE, 2009), entre otros, analizan la CP desde un enfoque sintáctico, esto es, describen las oraciones pasivas como construcciones marcadas, donde el verbo toma una forma particular y el paciente toma el lugar del agente, que se transforma en término oblicuo o desaparece. Para Mendikoetxea, las construcciones pasivas (derivadas de la activa) están dentro de la clase general de las construcciones inacusativas. En cuanto al significado de la CP, esta autora señala que al ser el objeto nocional el punto de partida de la construcción, el objeto cumple las funciones discursivas de tema y tópico. Por su parte, la RAE-ASALE señala que las oraciones pasivas son estructuras intransitivas que se forman con verbos transitivos. El paciente de la acción expresada que corresponde al complemento directo pasa 
a ser sujeto de la oración pasiva (2009: \$41.2a). Es decir, se estudian las CP desde un punto de vista sintáctico y se analizan como derivadas de la voz activa. Asimismo, se advierte que las oraciones pasivas expresan el mismo contenido que las activas correspondientes (es decir, que tienen el mismo significado), pero que se prefieren las pasivas a las activas "cuando se desea destacar al paciente, cuando no se posee información sobre el agente o cuando se desea mantenerlo en segundo plano” (2009: \$41.2f).

Más allá de los enfoques morfosintácticos, se encuentran los gramáticos que analizan la CP desde un enfoque semántico-psicológico: Lenz (1935: \$57 y \$253), Gili Gaya (1955: §56), RAE (1973: \$3.5.2/3) y Seco (1999: 187). Según este enfoque, el hablante tiene interés por el objeto/paciente.

Finalmente, Alarcos Llorach (1966: \$3), en contraposición con el resto de las gramáticas, considera que no existe la voz pasiva en español. En este sentido, ejemplos como Juan fue vencido y Juan fue vencedor son estructuras atributivas. Para este autor, el sentido pasivo resulta sólo del contenido total de la oración sin presentar manifestación morfológica y/o sintáctica de ningún tipo.

\subsubsection{Estudios no hispánicos}

Desde diversas teorías gramaticales, pueden advertirse tres grandes interpretaciones sobre la voz pasiva. La primera concibe la voz pasiva como la promoción del objeto y consecuente democión del sujeto a caso oblicuo (por ejemplo, Brucart, 1990: 195). Una segunda interpretación es la que plantea la voz pasiva como estrategia de topicalización del 
paciente (Givón, 1979). ${ }^{4}$ En tercer y último lugar, se encuentra la tesis de Shibatani (1985) de que la voz pasiva no es una mera topicalización del paciente sino que es una defocalización del agente.

\subsubsection{La propuesta de Borzi desde el ECP}

Desde el ECP, Borzi parte de la premisa de que la construcción pasiva (CP) es una estructura en sí misma y no derivada (Borzi, 2000; 2004; 2005). Dentro de las CP, no todas tienen el mismo significado. La autora distingue las construcciones pasivas con ser (Cpser) y las construcciones pasivas de pronombre se (Cpse). La Cpser, según Borzi, es la gramaticalización de una zona intermedia entre el modelo cognitivo idealizado ${ }^{5}$ de bola de billar y el modelo cognitivo idealizado de escenario, ${ }^{6}$ donde el objetivo comunicativo del hablante es describir el desarrollo del cambio en el paciente, es decir, cómo opera en el lugar del cambio la energía transmitida (la pasiva de ser no hace hincapié ni en el

\footnotetext{
4 "Passivization is the process by which a non-agent is promoted into the role of a main topic of the sentence. And to the extent that the language possesses coding properties which identify main topics as subjects and distinguish them from topics, then this promotion may also involve subjectivization" (1979: 186). ${ }^{5}$ Para el ECP, entre los principios generales de organización de la cognición se encuentran los Modelos Cognitivos Idealizados (MCI), mediante los cuales se organizan los conocimientos. Al respecto, Lakoff (1987) postula que los hablantes organizan el conocimiento mediante estructuras de MCI, que son los sostenes de las categorías entendidas en términos graduales o no graduales.

${ }^{6}$ Desde el ECP, existen dos modelos cognitivos idealizados (MCI) que están en la base de la conceptualización de los nombres y de los verbos: el MCi de escenario, donde se objetiviza la interacción de un participante con una región; y el MCI de bola de billar o cadena de acción, donde los objetos físicos discretos se mueven e interactúan energéticamente cuando entran en contacto (Langacker, 1991).
} 
traspaso, ni en el resultado, sino en el proceso del cambio). El paciente goza así (como en el modelo de escenario) de alta autonomía conceptual. Equilibrando esto, el agente no está en la mira, pero sigue vigente (ejemplo: El sospechoso fue detenido a las 0.45 por efectivos de la comisaría $3^{a} y$ de la División Robos y Hurtos de la Policía Federal en Barracas) (Borzi, 2004: 4-5). En tanto, el hablante elige la Cpse (en lugar de la Cpser) para destacar una figura contra un fondo en un proceso de intransitivización que elige el paciente como pivote, y en ese sentido, se acerca al modelo de escenario (ejemplo: En Roger Park se registraron cuatro tiroteos en calles distintas) (Borzi, 2005: 18-19). Borzi comprueba sus hipótesis mediante el análisis de un corpus escrito compuesto de artículos periodísticos de la ciudad de Buenos Aires.

Teniendo en cuenta lo dicho previamente, en el presente trabajo consideraremos construcción pasiva a una construcción en sí misma, con significado propio, no derivado de la activa (siguiendo a Borzi), caracterizada como una predicación cuyos participantes tienen atributos propios.

\subsection{El complemento agente}

Es de destacar que no todas las gramáticas hispánicas describen el complemento agente (CAg). Entre las que lo mencionan, se encuentran la GRAE de 1931 (\$275) y el Esbozo (1973: \$3.5.2.), que dividen las oraciones pasivas en primeras y segundas, según aparezca o no el agente (en las primeras se explicita el CAg, mientras que en las segundas, no). Además, concluyen que en el CAg se usa más por que de, debido a que consideran el agente como medio o instru- 
mento de acción, y no como punto de origen de esta (1931: $\$ 275)$. Gili Gaya (1955: \$193) advierte que el uso de de como agente de pasiva ha desaparecido casi enteramente. Kovacci (1990-1992) también advierte que el agente se construye generalmente con la preposición por, aunque también acepta de (1990-1992: \$5.2.5).

Desde el generativismo, De Miguel (1992) señala que el CAg no siempre es agente: en oraciones como la población está rodeada por montañas, a menudo se ha llamado complemento agente al sintagma-por (en términos generativistas), aunque, desde un punto de vista semántico, no tiene por qué ser agente. En efecto, De Miguel señala que el sintagmapor puede ser una meta (los libros fueron recibidos por el periodista) o un tema (el prado está limitado por la valla). La autora añade que, desde el punto de vista sintáctico, está en discusión si el CAg es un adjunto o un argumento (discusión que no tiene lugar desde el ECP, ya que no hablamos de estructura argumental ni de marco casual sino de esquemas verbales con verbos y actantes). ${ }^{7}$

Mendikoetxea (1999: \$25.4.2.1.) afirma que en la perífrasis con estar, el elemento locativo es el sujeto sintáctico y el otro argumento verbal se expresa por medio de un sintagma preposicional introducido por de o por: En La ciudad está rodeada de montañas, la construcción de montañas no sería CAg sino simplemente un sintagma preposicional.

Bosque (1999: \$4.4.5.1) también advierte que la gramática de los CAg es más compleja en el caso de las CP con

\footnotetext{
${ }^{7}$ Véase Borzi (2008) para una explicación más detallada de los esquemas verbales.
} 
estar que en el de las de ser. Sobre todo con verbos que el autor denomina transitivos estativos, como rodear, ocupar, sitiar, cercar, formar, habitar, constituir, integrar, entre otros. Para Bosque, en las construcciones con estar no habría CAg sino complementos pseudoagentivos. Cabe destacar que Bosque es de los pocos autores que prestan especial atención a la preposición introductora del CAg. El gramático español señala que por es la preposición no marcada, de uso más general en la lengua actual. La preposición de, en tanto, se emplea en la actualidad de forma mayoritaria en las construcciones estativas. Al respecto de de, Bosque clasifica el uso de esta preposición según el tipo de construcción estativa: a) predicados de afección: (aborrecido de todos); b) predicados estativos que indican forma, posición y locación (vive rodeado de enemigos); c) en la lengua antigua, más raramente en la actual, los complementos con la preposición de incidían también sobre predicados de influencia (de Dios seas perdonado) (1999: \$4.4.5.1.).

Finalmente, la RAE-ASALE postula que la alternancia de las preposiciones por y de está relacionada con las propiedades léxicas de los participios, y no tanto con la naturaleza gramatical de las oraciones pasivas (2009: $\$ 27.8$ p).

Desde el ECP y afines, ciertos autores han estudiado más en profundidad la función de CAg. Así, Miñones y Sánchez (2000) analizaron la CP de frase verbal con ser en el discurso narrativo (tomando un libro de cuentos: Al Capitán de los duendes) y en el discurso histórico (tomando el texto La República perdida) y concluyeron que los atributos del agente introducido por la preposición por son: [+voluntad] y [+humano], en contextos con pacientes altamente afectados de 
atributos [+determinado], [+individual] y [-humano]. Las autoras no contemplaron en su estudio el CAg introducido por de.

Por su parte, Delbecque $(2003)^{8}$ analiza la alternancia de las preposiciones por, de, con y en en el CAg. En cuanto a la preposición por, esta autora plantea que suele denotar entidades animadas y colectivas y aparece típicamente en construcciones participiales y pasivas auxiliadas (2003: 10). En relación con la preposición de, Delbecque plantea que en general esta preposición introduce estados de cosas, eventos y estados de ánimo, es decir, entidades de contornos más bien inespecíficos, vagos, que se sitúan en una relación de continuidad con la entidad sujeto (2003: 9).

En último lugar, cabe destacar un estudio reciente, enmarcado en un enfoque diacrónico. Se trata del trabajo de Nieuwenhuijsen (2013). La autora estudia la frecuencia y

\footnotetext{
${ }^{8}$ En su estudio, Delbecque distingue siete formas de predicado con significado pasivo: 1. Pasiva auxiliada, es decir, compuesta de un auxiliar seguido de un participio pasado: esto incluye los verbos ser y estar ("sus vidas fueron fracturadas y avasalladas por una orden que usted dio”); 2 . Construcción participial adnominal ("los miembros de las juventudes comunistas, de manos empuñadas, rodeados de soldados con ametralladores, empezaron a cantar la Internacional”); 3. Pasivas reflejas ("No habiendo ocurrido ni lo uno ni lo otro, las soluciones democráticas deben derivarse de las proposiciones democráticas"); 4 . Construcciones medias ("Europa puede beneficiarse de esta dinámica regional favorable"); 5. Causativas reflejas, es decir, los casos en los que el clítico reflexivo se asocia con un auxiliar causativo seguido de un infinitivo ("Nosotros no podíamos (...) dejarnos llevar por nuestras emociones"); 6. Pasivas reflejas auxiliadas, esto es, los sintagmas verbales que contienen a la vez un clítico reflexivo y un auxiliar con participio pasado ("Su padre era un ganadero de origen cántabro que cuidaba vacas, pero que se sintió atraído por la cuidad”); 7. Predicación secundaria, con un participio que predica al complemento de objeto directo ("el Ejecutivo no posee una mayoría en el Senado y continuamente ve sus proyectos vetados por la oposición”) (2003: 6-8).
} 
distribución de las preposiciones de y por en construcciones pasivas con ser a lo largo de la historia del español en un corpus digital diacrónico del siglo XIII al xx (Corpus de español).

Nieuwenhuijsen advierte que la preposición de era originalmente la preposición más frecuente, mientras que por era una forma minoritaria hasta que la desplazó. La autora describe el proceso de desplazamiento (los nuevos verbos), pero no estudia las razones de dicho desplazamiento.

Los estudiosos de la historia de la lengua afirman que la preposición $d e$, que tiene su origen latino en la propia de latina, puede deber su uso agentivo en el significado de denotar que la acción se originaba en o partía de determinada persona o cosa. También podían tener un valor instrumental. En contraste, por tiene su origen en la preposición latina per, cuyo significado se relacionaba con que la acción tenía lugar o se ejecutaba a través de cierta persona o cosa. Keniston (1937: 472), para el siglo XVI, observa que se utiliza de cuando la acción es indeterminada, en tanto que por aparece con verbos que expresan una acción determinada (perfectiva) y agentes igualmente determinados. Una distribución parecida encuentra Ricós Vidal (1988-1989: 185186).

Luego del extenso análisis cuantitativo diacrónico, Nieuwenhuijsen concluye que existen tres fases de propagación de por: a) por minoritaria hasta el siglo XIII; b) alternancia estable de de y por con frecuencias parecidas en los siglos XIV a XVIII inclusive; c) generalización de por (se vuelve la preposición no marcada) en los siglos xIX y Xx. Esta evolución cumple con el ritmo de slow-quick-quick-slow observado en 
muchos otros cambios lingüísticos, además de que muestra la típica curva S, si bien de forma más alargada (2013: 13).

Queda sin discusión el motivo por el cual ocurrió este cambio sintáctico, este desplazamiento de de a por. En este sentido, la lingüista sólo repite a Herrero Ruiz de Loizaga, quien dice: "la mayor nitidez de esta preposición para indicar el agente de un modo más claro que la preposición de, utilizada para múltiples relaciones sintácticas, nexo, por lo tanto, menos preciso" (1992: 355). Herrero tampoco explica demasiado, sino que se apoya en la polisemia de de, en contraste con la especificidad mayor de por.

En el presente trabajo veremos cómo de se especializa en contextos estáticos (mientras que por aparece en contextos dinámicos), lo que justifica su elección, porque el significado básico de de es el de posesión, una relación estática.

Considerando todo lo anteriormente reseñado, en el presente estudio se entenderá la noción de complemento agente como la construcción que manifiesta al actante ${ }^{9}$ agente en una predicación con sentido pasivo. Se tomará el término agente en un sentido convencional, ya que se tiene en cuenta la escala de agentividad, esto es, partiendo de la cláusula transitiva prototípica, el actante agente presenta una escala

\footnotetext{
${ }^{9}$ Desde el ECP, la noción de actante (lo que para otras teorías serían argumentos, papeles temáticos o semánticos, o casos) se refiere a "entidades móviles, cambiantes en todos y en cada uno de sus atributos que se redefinen cada vez en cada mensaje al combinarse con cada verbo y con cada otro actante en un contexto determinado. Es decir, que así como no hay verbos en tanto lexemas fijos con características fijas, tampoco hay actantes preexistentes de características fijas y obligatorias, tanto en la ausencia/presencia de esas características como en el grado de cada una de esas características. Es cada una de las combinatorias las que definen a cada uno de los elementos que componen el evento" (Borzi, 2008: 1).
} 
desde el más prototípico hasta el más marginal: agente (Juan pinta la pared), experimentante (Juan piensa), benefactivo (Juan tiene frío), instrumental (La piedra rompió el vidrio), tema (El lápiz está sobre la mesa).

\section{Los problemas pendientes}

Ninguno de los teóricos mencionados caracteriza el CAg semánticamente, esto es: no explican el motivo de su aparición; no describen semánticamente el actante en sí. Eventualmente, atienden a la preposición que lo introduce. Coinciden en que por es la de mayor frecuencia, evocan la ocasional presencia de $d e$, aunque no se valida la afirmación con datos empíricos. De los estudios aquí reseñados, sólo los trabajos de De Miguel, Mendikoetxea y Bosque intentan una caracterización semántica del CAg. Advierten, por ejemplo, que no siempre se trata de agentes y que tienen un comportamiento distinto según se construyan con el verbo ser o estar. Sin embargo, ilustran sus afirmaciones con ejemplos ad hoc que no tienen realidad concreta en el uso de la lengua, como el varias veces citado ejemplo la población está rodeada por montañas, donde la concurrencia de rodear con la preposición por no suele darse en la lengua actual, como se refleja en el corpus analizado en el presente trabajo. El problema, insistimos, está en la falta de validación de las hipótesis con datos empíricos.

Los autores que analizan la CP desde enfoques funcionalistas o cognitivistas advierten que existen diferencias sustanciales entre los participantes de la cláusula activa y los de la cláusula pasiva. Sin embargo, no hay acuerdo absoluto 
acerca de cuáles son los rasgos más típicos de los participantes del evento pasivo. No se describe la (posible) diferencia sintáctico-semántica entre los CAg introducidos por diferentes preposiciones.

Teniendo en cuenta estos problemas pendientes, el presente trabajo espera aportar evidencia empírica a la caracterización de las construcciones pasivas con CAg introducidos por por y por de. Se analizará el cuerpo de datos oral PRESEEA-Buenos Aires. Dada la escasez de ejemplos, se completará el estudio con un corpus escrito del año 2014.

\section{La propuesta}

\subsection{Antecedentes}

En trabajos previos (Funes, 2011, 2012) se caracterizó en el Habla culta de la ciudad de Buenos Aires el CAg introducido por la preposición de en su contexto preferido de aparición, comparándolo, en un primer análisis, con la cláusula transitiva prototípica, y en un segundo estudio, con el CAg introducido por la preposición por. Se extrajeron las siguientes conclusiones:

i) En corpus oral se constata un uso marginal de construcciones pasivas y en particular del CAg introducido por la preposición de.

ii) El hablante tiende a elegir la preposición de para acompañar agentes con los atributos [-humano] y [-determinado], en contextos de pacientes con baja afectación, y en esquemas de predicaciones con entidades estáticas preexistentes. 
iii) El hablante tiende a elegir la preposición por en contextos con pacientes altamente afectados y con agentes [+humanos] y [+determinados], en esquemas de predicaciones de eventos dinámicos.

Sobre corpus escrito, se concluyó:

i) Hay mayor frecuencia de CP, en relación con los corpora orales analizados.

ii) Dentro de las CP, hay mayor frecuencia de aparición de CAg introducidos por por, en contraste con los introducidos por de.

iii) Cuando el hablante elige la preposición por para introducir un CAg, lo hace para acompañar agentes con los atributos [+humano] y [+determinado], en contexto de sujetos pacientes con media o alta afectación, y en esquemas de predicaciones dinámicas (manifestadas en $\mathrm{CP}$ de frase verbal con ser, en su mayoría).

iv) En contraste, cuando el hablante elige la preposición de para introducir un CAg, lo hace para acompañar agentes con el atributo [-humano] y [-determinado], en contextos de sujetos pacientes con baja afectación, y en esquemas de predicaciones con entidades estáticas (manifestadas mayormente por predicaciones no finitas).

Las conclusiones extraídas del análisis en corpus escrito corroboran los resultados derivados del análisis en corpora orales (a excepción del tipo de estructura sintáctica predominante para ambas preposiciones). 


\subsection{Hipótesis}

Considerando los resultados previos, el presente trabajo espera aportar evidencia empírica a las hipótesis ya propuestas y resumidas en el apartado 3.1, reforzando el análisis con una hipótesis sobre la jerarquía de los atributos. Se sostiene que en la elección de una u otra preposición para introducir el CAg, el hablante considera sobre todo el tipo de predicación en la que está inmersa la construcción pasiva. En el caso de la preposición por, el hablante la elige en contextos de predicaciones dinámicas, cercanas al MCI de bola de billar, donde se observa la correlativa alta afectación del paciente. En contraste, el hablante elige la preposición de en contextos de predicaciones estáticas, con pacientes poco afectados. El hecho de que la preposición de sea elegida en las predicaciones estáticas se relaciona con el significado básico que tiene $d e$, que es el de posesión, tal como se propone en Funes (2015). En este último estudio, siempre en el marco teórico del Enfoque Cognitivo Prototípico, se proponía el análisis pragmático, semántico y sintáctico de la preposición de en contexto nominal, en el español de Buenos Aires. En cuanto a la caracterización semántica, el trabajo concluía que la preposición de presentaba una estructura de categoría radial (Lakoff, 1987), cuyo significado central era el de posesión. Este significado básico se combina con el de los elementos que la rodean para establecer los significados restantes. Las hipótesis de ese trabajo se validaron con un análisis cualitativo y cuantitativo, sobre el corpus Habla culta de la ciudad de Buenos Aires, que recoge usos de la década de 1960 (también se analizó el corpus oral PRESEEA-Buenos 
Aires como corpus control, ya que recoge usos actuales, de los años 2010-2011).

El significado básico de la preposición de, entonces, es el de posesión, que designa una relación estática. La relación de posesión se establece entre dos actantes, el poseedor y el poseído. Es una relación cuyo punto de partida es el poseedor y desde allí se identifica el elemento poseído. En la posesión prototípica, la caracterización de los actantes (el poseedor y el poseído) se realiza sobre los atributos propuestos para los participantes de la cláusula transitiva prototípica (Hopper y Thompson, 1980). En este sentido, el poseedor prototípico se asemeja al agente en algunos atributos: [+humano], [+voluntad] y [+punto de partida], [+individualizado], [+determinado]. ${ }^{10}$ En contraste, el poseído prototípico presenta algunos atributos semejantes a los del paciente: [+concreto], [-humano], [-voluntad], [+inanimado], [+compacto], [+específico], [+delimitado], [+contable], [+individual], [-abstracto]. ${ }^{11}$

En cuanto a la relación en sí misma, la posesión prototípica establece una relación estática, ya que no hay traspaso de energía de un actante hacia el otro; es exclusiva, es decir, el poseedor es uno solo. Además, el poseedor tiene derechos sobre el poseído, en el sentido de ejercer cierto dominio sobre él, tiene responsabilidad sobre lo poseído (se espera que

\footnotetext{
${ }^{10}$ Se diferencia en otros, ya que el poseedor no tiene los atributos [+ejecutor] ni [+iniciador] o [+fuente de energía], debido a que no participa de un evento dinámico de desplazamiento de energía hacia un actante paciente, sino que forma parte de una relación estática.

${ }^{11}$ A diferencia del paciente prototípico, el poseído no presenta afectación, ya que no sufre el desplazamiento de energía proveniente de un agente, en tanto no participa de un evento dinámico.
} 
lo mantenga en buenas condiciones). La relación de posesión prototípica es de largo plazo (se extiende en el tiempo, y tiene un inicio) y exige que haya cercanía espacial entre poseedor y poseído.

En las construcciones pasivas con CAg, de aparece cuando las predicaciones son estáticas (cercanas al MCI de escenario), de la misma manera que en las construcciones nominales aparece para designar una relación estática de posesión. En tanto, por se especializa en predicaciones dinámicas, donde hay más interacción entre agente y paciente.

\section{Metodología y corpus}

Se realizó un análisis cuantitativo y cualitativo sobre dos cuerpos de datos auténticos: el corpus oral PRESEEA-Buenos Aires, ${ }^{12}$ que recoge usos de la ciudad de Buenos Aires de los años 2010-2011 y un corpus escrito de 51 notas periodísticas del mes de julio de 2014, pertenecientes a los diarios de mayor tirada de la ciudad de Buenos Aires. Se midieron los siguientes atributos en los actantes agente y paciente: [+/-humano], [+/-determinado]; se midió, además, el grado de afectación del paciente, teniendo en cuenta la jerarquía de subtipos propuesta por Borzi (2004). ${ }^{13}$ Finalmente,

\footnotetext{
${ }^{12}$ El PRESEEA es el Proyecto para el Estudio Sociolingüístico del Español de España y de América. El de Buenos Aires recoge usos de los años 2010 y 2011, de distintos niveles socioculturales y etarios. El corpus está conformado por 12 entrevistas semidirigidas con grabador a la vista.

${ }^{13}$ De acuerdo con esta jerarquía, el 'paciente locativo' sería el que sufre menos afectación, seguido del 'paciente objeto'. En el otro extremo de la escala, presentando alta afectación, estaría el 'paciente factitivo', seguido del 'paciente cambio de estado'.
} 
se midió el tipo de predicación (dinámica o estática) de la que formaba parte el CAg.

\section{Análisis. Resultados y discusión}

A continuación, se examinan cualitativamente algunos ejemplos de cada preposición para ver cómo operan los atributos mencionados en un contexto auténtico de uso. Se presentan primero ejemplos de por en el corpus oral PRESEEA. Se destaca en negrita la cláusula pasiva bajo análisis:

(1) -En una época, ya tenía, ya habían nacido mis dos hijos, y estábamos bastante agobiados por los costos de mantenimiento de un departamento que no era grande ni era tan lujoso, donde vivíamos (Entrevista 3, p. 8).

(2) 11. E.: ¿La comida quién la preparaba?

I.: Habitualmente, las mujeres mayores, ¿ $\mathrm{mmm}$ ?, era preparada por ellas (Entrevista 9, p. 8).

En el ejemplo (1), aparecen un agente [-humano], [+determinado] y un paciente [+humano], [-determinado], con alta afectación, en el contexto de una predicación dinámica. La idea es que los costos del departamento agobian a sus habitantes. El agobio provoca alta afectación y se conceptualiza como el MCI bola de billar: un elemento transmite energía y causa un cambio en un paciente. Este resto de dinamismo evocado por el participio "agobiado" contrasta con la semántica del verbo estar. En (2) encontramos un ejemplo más típico: agente [+humano], paciente [-humano] (ambos 
determinados), predicación dinámica manifestada en una pasiva con ser. Los dos ejemplos son distintos en cuanto al tipo de construcción (estar + participio en contraste con ser + participio) y en cuanto a los atributos de los actantes, pero tienen en común la conceptualización dinámica, dada sobre todo por el participio y por la afectación del paciente.

A continuación, se observan dos ejemplos de construcciones pasivas con la preposición de:

(3) I.: Sí, yo soy tan apasionado de lo que hago que en realidad me... a veces eh... me olvido de poner mi capacidad al servicio de una un bienestar económico que que por ahi quisiera tener (Entrevista 1, p. 3).

(4) 3. E.: ¿Notaste algún cambio que haya sufrido tu barrio desde que vivís ahí?

I.: Y... se puso un poco más comercial, yo estoy sobre una avenida, que si bien es más tranquila que otras avenidas, es avenida al fin, y se pobló de negocios, por ejemplo, que no estaban cuando yo me mudé (Entrevista 9, p. 4).

Los ejemplos (3) y (4) presentan predicaciones más estáticas, dado que en el primer ejemplo se ve una atribución del que habla (es apasionado) y en el segundo ejemplo, la visión resultativa de un barrio que pasó de ser residencial a tener muchos negocios. En (3) conviven un sentido atributivo con un sentido pasivo, en tanto es la profesión del entrevistado lo que lo apasiona (podría parafrasearse como "lo que hago me apasiona”, donde la profesión, agente [-humano] y [+determinado] transmite energía y provoca un cambio 
en el paciente "me" [+humano] y [+determinado]). En (4), encontramos una pasiva con se, que raramente acepta la presencia de CAg. En la construcción, hay un paciente locativo ("el barrio") y un agente [-humano] y [-determinado] ("negocios").

Considerando, entonces, la preposición introductora de CAg, la diferencia más notoria en los ejemplos presentados es el tipo de predicación: más dinámica con la preposición por, y más estática con la preposición de. Aquí se confirma que el atributo de más peso es el de tipo de predicación, por sobre los atributos de los actantes.

En el análisis cuantitativo, ilustrado en el cuadro I, se advierten los escasos ejemplos de construcciones pasivas que ocurren en discurso oral.

\section{Cuadro I. Atributos de las construcciones} pasivas en corpus oral

\begin{tabular}{|l|r|r|}
\hline \multicolumn{1}{|c|}{ Atributos } & CAg por & CAg $\boldsymbol{d e}$ \\
\hline Agente [+humano] & 7 & 0 \\
\hline Agente [-humano] & 3 & 3 \\
\hline Agente [+determinado] & 7 & 0 \\
\hline Agente [-determinado] & 3 & 3 \\
\hline Paciente [+humano] & 2 & 1 \\
\hline Paciente [-humano] & 8 & 2 \\
\hline Paciente [+determinado] & 7 & 2 \\
\hline Paciente [-determinado] & 3 & 1 \\
\hline Predicación dinámica & 6 & 0 \\
\hline Predicación estática & 4 & 3 \\
\hline Grado de afectación alto del paciente & 5 & 0 \\
\hline Grado de afectación medio del paciente & 1 & 0 \\
\hline Grado de afectación bajo del paciente & 4 & 3 \\
\hline Total & $\mathbf{1 0}$ & $\mathbf{3}$ \\
\hline
\end{tabular}


Las pocas ocurrencias de construcciones pasivas no permiten sacar conclusiones definitivas. Sólo podemos comentar que la preposición por muestra una leve tendencia a introducir agentes [+humanos] y [+determinados], en combinación con pacientes [-humanos] y [+determinados], y siempre ocurre en contextos de predicaciones dinámicas, con la consecuente alta afectación del paciente. En contraste, la preposición de muestra una leve tendencia a introducir agentes [-humanos] y [-determinados], y siempre ocurre en contextos de predicaciones estáticas, con la consecuente baja afectación del paciente. A su vez, los atributos del paciente muestran oscilación, entre [+/-humanos] y [+/-determinados].

Para complementar el análisis y poder constatar si estas tendencias observadas en el corpus oral se repetían en el corpus escrito, se analizaron ejemplos de 51 notas periodísticas. En cuanto al análisis cualitativo, se examinan, en primer lugar, los siguientes ejemplos de CAg introducidos por la preposición por:

(5) Sincera y dolida

Contó por qué se sintió maltratada por él, y reveló que él la tenía agendada en su celular como "Lucifer 666".

(Clarín, martes 22 de julio de 2014)

(6) Destino, casualidad o milagro. Por minutos o una decisión de último momento, varias personas salvaron sus vidas porque decidieron no abordar el vuelo que fue derribado por un misil en Ucrania y que provocó la muerte de 298 personas.

(Clarín, viernes 18 de julio de 2014) 
(7) Con cada vez menos visitantes, crece el abandono en el cementerio.

Espacio público. Las galerías de nichos están destrozadas por el agua y la suciedad. $\mathrm{Y}$ afuera quedan muy pocos vendedores de flores.

En los ejemplos (5) al (7) se observa claramente la alta afectación del paciente, dada por el significado del participio y el dinamismo de la predicación (dinamismo en el sentido de poder recuperar un MCI de bola de billar). En (5), el agente [+humano] y [+determinado] se combina con un paciente [+humano] y [+determinado], en un contexto de predicación dinámica (en construcción de participio). En (6), observamos una construcción pasiva con ser, donde el agente es [-humano], pero en realidad se trata de una metonimia, ya que detrás del misil está el verdadero agente humano que lo lanzó. El paciente es [-humano] ("el vuelo"), pero la alta afectación recae sobre los seres humanos que eran pasajeros de ese vuelo. En (7) vemos que hay alta afectación, pero dada por un agente causa ("el agua" y "la suciedad") a un paciente [-humano] ("galerías de nichos"). La presencia del verbo estar obedece a que se conceptualiza el resultado: el agua y la suciedad destrozaron las galerías de nichos con el paso del tiempo, y lo que vemos hoy en día es la consecuencia ("destrozadas"). Los tres ejemplos comparten la alta afectación del paciente y el dinamismo de la predicación, aunque en (4) sea una construcción participial y en (7), resultativa.

A continuación, se observan algunos ejemplos de CAg introducidos por de: 
(8) La velaron rodeada de alcohol y cigarrillos

La casa velatoria encargada del funeral, en Lousiana, hizo caso a los pedidos de las hijas de Burbank, quienes querían que la última despedida de su madre reflejara su manera de vivir. (http://www.lmcordoba.com.ar/nota.php?ni=169572)

(9) La 'selfie' más tierna del clan Tinelli: Lorenzo rodeado de amor (http://www.pronto.com.ar/articulo/famosos/selfie-mas-tiernaclan-tinelli-lorenzo-rodeado amor/20140721140311146264.html)

(10) Pasó sus últimas horas rodeado de sus amigos

(http://www.perfil.com/protagonistas/Paso-sus-ultimas-horasrodeado-de-sus-amigos-20140412-0033.html)

Los ejemplos anteriores ocurren con el mismo verbo (rodear), no considerado por Nieuwenhuijsen en el estudio citado. Los agentes son [-humanos] y [-determinados], excepto en (10) (agente [+humano] y [+determinado] "sus amigos"). Los pacientes son [+humanos] y [+determinados]. En los tres ejemplos, se observa la baja afectación del paciente, y la predicación estática (más cercana al MCI de escenario) en construcciones de participio.

En cuanto al análisis cuantitativo, observamos el número de ocurrencias y la distribución de los atributos en el cuadro II: 
Cuadro II. Atributos de las construcciones pasivas en corpus escrito

\begin{tabular}{|l|r|r|}
\hline \multicolumn{1}{|c|}{ Atributos } & CAg por & CAg $\boldsymbol{d e}$ \\
\hline Agente [+humano] & 23 & 1 \\
\hline Agente [-humano] & 21 & 5 \\
\hline Agente [+determinado] & 30 & 0 \\
\hline Agente [-determinado] & 14 & 6 \\
\hline Paciente [+humano] & 10 & 6 \\
\hline Paciente [-humano] & 34 & 0 \\
\hline Paciente [+determinado] & 30 & 5 \\
\hline Paciente [-determinado] & 14 & 1 \\
\hline Predicación dinámica & 34 & 0 \\
\hline Predicación estática & 10 & 6 \\
\hline Grado de afectación alto del paciente & 28 & 0 \\
\hline Grado de afectación medio del paciente & 10 & 0 \\
\hline Grado de afectación bajo del paciente & 6 & 6 \\
\hline Total & $\mathbf{4 4}$ & $\mathbf{6}$ \\
\hline
\end{tabular}

En primer lugar, se observa con claridad la mayor frecuencia de ocurrencias de CAg con por (44 casos), por encima de los CAg con de (tan sólo 6 casos). Se constata la tendencia de que la preposición por participe de predicaciones dinámicas, mientras que de se especializaría en predicaciones estáticas (sin preferir ningún tipo de construcción sintáctica por sobre otra: es decir, encontramos construcciones pasivas con verbo ser, con verbo estar y construcciones de participio). El segundo atributo que resulta más importante es el de la afectación del paciente, en consonancia con el tipo de predicación. En las conceptualizaciones más cercanas al MCI de bola de billar, se observa una alta afectación del paciente (como ocurre en la cláusula transitiva prototípica). Por su parte, en las predicaciones estáticas, más cer- 
canas al MCI de escenario, la afectación del paciente es baja o prácticamente nula. Los atributos de los actantes, si son [+/-humanos] o [+/-determinados] no parecen incidir en la elección de una u otra preposición de manera definitoria, aunque sí se observa una leve tendencia de aparición de agentes [-humanos] y [-determinados] introducidos por la preposición de. Estos atributos se relacionan a su vez con el tipo de predicación: la tendencia de de a acompañar agentes [-humanos] y [-determinados] (como en "rodeado de amor" o "rodeada de alcohol y cigarrillos") obedece a que son actantes que no están ejecutando acciones sino describiendo una situación. Para el papel de agente prototípico que transmite energía a un paciente, el atributo [+humano] es el más frecuente, como ocurre en "maltratada por él", aunque en los ejemplos encontremos agentes metonímicos (como "un misil") y agentes causa, es decir [-humanos], como "el agua y la suciedad". Al ser actantes identificados, la tendencia es que sean [+determinados]. En cuanto al actante paciente, en las predicaciones dinámicas de por, el paciente tiende a ser [-humano] y [+determinado], como sucede con el paciente de la cláusula transitiva prototípica. El tipo de predicación dinámica es el que suele estar acompañado de agentes [+humanos] y su contrapartida, paciente [-humano]. Finalmente, con la preposición de aparecen pacientes [+/-humanos] y [+determinados], aunque la escasez de ejemplos no permite afirmar que realmente esta sea la tendencia. Por lo pronto, los atributos del paciente, en los CAg introducidos por de no tendrían peso en la elección de esta preposición.

En ambos cuerpos de datos, se verifica una muy escasa frecuencia de uso de la preposición de para introducir CAg, 
tal como afirmaban las gramáticas mencionadas en el estado de la cuestión. Ahora bien, los pocos casos que encontramos deben ser explicados. Existen motivaciones para la elección de de sobre por. El hecho de que la preposición de aparezca en predicaciones estáticas y con pacientes con baja o nula afectación se relaciona con el significado prototípico de la preposición de, que es el de posesión (Funes, 2015). Recordemos que la relación posesiva es estática, ya que no supone traspaso de energía sino una visión estática de la situación.

En el cuadro III puede observarse una síntesis y recapitulación de las condiciones de la alternancia entre CAg introducidos por de y CAg introducidos por la preposición por:

\section{Cuadro III. Características de CAg introducidos por de y por}

\begin{tabular}{|l|l|l|}
\hline \multicolumn{1}{|c|}{ Parámetro } & CAg introducido por $d e$ & \multicolumn{1}{c|}{ CAg introducido por por } \\
\hline Tipo de agente & $\begin{array}{l}\text { [-humano } ; ; \\
{[\text {-determinado }]}\end{array}$ & $\begin{array}{l}\text { [+humano } /[\text {-humano }] ; \\
{[+ \text { determinado }]}\end{array}$ \\
\hline Tipo de paciente & $\begin{array}{l}{[+ \text { humano }] /[\text {-humano }] ;} \\
{[+ \text { determinado }]}\end{array}$ & $\begin{array}{l}{[\text {-humano }] ;} \\
{[+ \text { determinado }]}\end{array}$ \\
\hline Tipo de predicación & Estática & Dinámica \\
\hline $\begin{array}{l}\text { Grado de afectación } \\
\text { del paciente }\end{array}$ & Bajo & Alto \\
\hline
\end{tabular}

De este modo, puede ilustrarse, a partir de los siguientes esquemas, la motivación de la elección de por o de para introducir CAg en construcciones pasivas. En el Esquema I observamos que la preposición por aparece en predicaciones dinámicas, cercanas al MCI de bola de billar. En el ejemplo (Se sintió maltratada por él), el agente "por él" transmite energía hacia el paciente (recuperado en la desinencia del 
verbo "sintió") provocando un cambio en él. La transmisión de energía está representada por la flecha en dirección al paciente, y el cambio se refleja en el punto negro, en contraste con el punto blanco del agente.

Se sintió maltratada por él

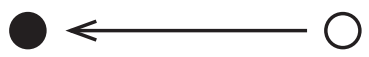

En el Esquema II, en tanto, observamos que la preposición de introduce CAg en predicaciones estáticas, cercanas al MCI de escenario. En el ejemplo (Lorenzo rodeado de amor), agente (amor) y paciente (Lorenzo) aparecen en una escena en la que no hay transmisión de energía sino solamente co-ocurrencia de ambos elementos en una misma situación. Al no haber energía, tampoco hay afectación en el paciente, lo que vemos reflejado en el punto blanco y en la línea que reemplaza a la flecha que se observaba en el Esquema I. El borde del esquema ilustra la conceptualización estática (a la manera de escenario) de la frase Lorenzo rodeado de amor: se trata de un bebé que disfruta del amor de sus seres queridos. No se conceptualiza un movimiento, sino una visión estática del evento.

Lorenzo rodeado de amor

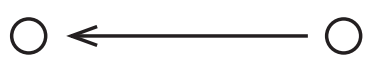




\section{Conclusiones}

A partir del análisis de los cuerpos de datos, se concluye que en corpus escrito hay mayor frecuencia de construcciones pasivas que en el corpus oral PRESEEA. En las construcciones pasivas con complemento agente explícito, hay mayor frecuencia de aparición de CAg introducidos por por, en contraste con los introducidos por de, de los que hallamos muy pocos casos.

Existe una jerarquía en los atributos de las construcciones pasivas, en cuanto a la elección de cada preposición. Los atributos que tienen más peso son el tipo de predicación y el grado de afectación del paciente, seguido de los atributos del agente. La caracterización del paciente no parece ser relevante en la elección de la preposición, según los datos analizados.

De esta manera, el hablante elige la preposición por para introducir un CAg en contexto de predicaciones dinámicas, es decir, con cercanía al MCi de bola de billar, mientras que se elige la preposición de para introducir un CAg en contextos de predicaciones estáticas. El hecho de que el tipo de predicación sea el atributo con más peso para la elección de una u otra preposición se debe a los significados básicos de dichas preposiciones. Es decir, el significado de por está relacionado con el dinamismo, movimiento o interacción entre los elementos, mientras que el significado de de se relaciona con conceptualizaciones más estáticas, en donde los elementos no interactúan o no presentan acciones que provoquen cambios. 


\section{Bibliografía}

Alarcos Llorach, E. (1966), "Pasividad y atribución en español", en Estudios de gramática funcional del espanol, Madrid, Gredos, pp. 163-171.

Alcina Franch, J. y J. M. Blecua (1975), Gramática española, Barcelona, Ariel.

Alonso, A. y P. Henríquez Ureña (1938-1939), Gramática castellana (2 vols.), Buenos Aires, Losada.

Bello, A. (1980 [1847]), Gramática de la lengua castellana, Madrid, EDAF.

Borzi, C. (2000), "La posición del paciente en la emisión de voz pasiva de frase verbal de ser + participio", en Gramática, discurso y sociedad (Actas de las I Jornadas de Lingüística "Beatriz Lavandera"), Buenos Aires, Universidad Nacional de Mar del Plata, 24 al 27 de marzo de 1999, pp. 47-57. (2004), La voz pasiva de ser + participio y los actantes en competencia, Signo y Seña 13, pp. 67-88.

(2005), "La función sintáctica 'sujeto pasivo'”, Anales del Instituto de Lingüística (Universidad Nacional de Cuyo), XXII y XXIII, pp. 7-40.

(2008), “Concepción de eventos y esquemas verbales".

Ponencia en el Congreso Internacional: Debates Actuales. Las teorías críticas de la Literatura y la Lingüística, Buenos Aires, agosto de 2008.

Bosque, I. (1999), "El sintagma adjetival. Modificadores y complementos del adjetivo. Adjetivo y participio", en I. Bosque y V. Demonte (dirs.), Gramática descriptiva de la lengua española, vol. 1, Madrid, Espasa Calpe, pp. 217310. 
Bosque, I. y V. Demonte (dirs.) (1999), Gramática descriptiva de la lengua española, Madrid, Espasa-Calpe.

Brucart, J. M. (1990), "Pasividad y atribución en español: un análisis generativo", en V. Demonte y B. Garza Cuarón (eds.), Estudios de lingüística de España y México, México, Universidad Nacional Autónoma de México, El Colegio de México, pp. 179-208.

Delbecque, N. (2003), "La variable expresión del agente en las construcciones pasivas", Nueva Revista de Filología Hispánica, LI, 2, pp. 373-416.

Funes, M. S. (2011), "La alternancia de las preposiciones por y de como introductoras del complemento agente", Revista de Estudios Lingüísticos de la Universidad de Alicante, 25, pp. 135-161.

(2012), "El complemento agente introducido por la preposición 'de'”, en G. Müller, L. Miñones y V. Barbeito (comps.), Estudios de lingüística cognitiva, Mendoza, Universidad Nacional de Cuyo, pp. 123-135.

(2015), Estudio sobre el uso de la preposición de en el español de Buenos Aires. Acercamiento desde el Enfoque Cognitivo-Prototípico, Múnich, LINCOM.

Gili Gaya, S. (1955), Curso superior de sintaxis española, Barcelona, Spes.

Givón, T. (1979), On understanding grammar, Nueva York, Academic Press.

Herrero Ruiz de Loizaga, F. (1992), "Algunas consideraciones en torno al complemento agente", Revista Española de Lingüística, 22, 2, pp. 339-359.

Hopper, P. y S. Thompson (1980), “Transitivity in grammar and discourse", Language, 56, 2, pp. 251-299. 
Keniston, H. (1937), The syntax of Castilian prose. The sixteenth century, Chicago, The University of Chicago Press. KovaccI, O. (1990-1992), El comentario gramatical I y II, Madrid, Arco Libros.

LAkoff, G. (1987), Women, fire and dangerous things, Chicago, The University of Chicago Press.

LANGACKER, R. (1987), Foundations of cognitive grammar. Theoretical prerequisites, vol. 1, Stanford, Stanford University Press.

(1991), Foundations of cognitive grammar. Descriptive applications, vol. 2, Stanford, Stanford University Press. LENZ, R. (1935), La oración y sus partes, Madrid, Nueva Revista de Filología Española.

MendikoetxeA, A. (1999), "Construcciones inacusativas y pasivas”, en I. Bosque y V. Demonte (dirs.), Gramática descriptiva de la lengua española, vol. 2, Madrid, Espasa Calpe, pp. 1575-1630.

Miguel, E. de (1992), El aspecto en la sintaxis del español; perfectividad e impersonalidad, Madrid, Universidad Autónoma de Madrid.

MiÑones, L. y K. Sánchez (2000), “Condiciones de aparición de la VpdeFV con 'ser' en el español escrito", en Gramática, discurso y sociedad (Actas de las I Jornadas de Lingüística "Beatriz Lavandera"), Buenos Aires, Universidad Nacional de Mar del Plata, 24 al 27 de marzo de 1999, pp. 1-12.

Nieuwenhuijsen, D. (2013), “En busca de la persona agente: la explotación de un corpus diacrónico digital para el estudio de las preposiciones de y por en construcciones pasivas", Scriptum Digital, 2, pp. 43-58. 
Real Academia Española (1931), Gramática de la lengua española, Madrid, Espasa Calpe.

__ (1973), Esbozo para una nueva gramática de la lengua española, Madrid, Espasa Calpe.

-ASAle (2009), Nueva gramática de la lengua española, Madrid, Espasa.

Ricós VidAL, A. (1988-1989), "Las construcciones pasivas en el español del siglo xv", Estudios de Lingüística, Universidad de Alicante, 5, pp. 183-189.

Rosetti, M. M. de (1969), "La frase verbal pasiva en el sistema español”, en A. M. Barrenechea y M. M. de Rosetti (eds.), Estudios de gramática estructural, Buenos Aires, Paidós, pp. 71-90.

SECo, M. (1999), Gramática esencial de la lengua española, Madrid, Espasa.

Shibatani, M. (1985), "Passives and related constructions: A prototype analysis", Language, 61, 4, pp. 821-848. 
